\title{
Uma Metodologia para Reduzir o Custo de Aprendizado para Técnicas Offline de Otimização de Aplicações Paralelas
}

\author{
Gustavo P. Berned ${ }^{1}$, Arthur Lorenzon ${ }^{1}$ \\ ${ }^{1}$ Laboratório de Otimização de Sistemas - Universidade Federal do Pampa \\ Campus Alegrete Caixa Postal 810 - 97546-550 - Alegrete - RS - Brasil \\ \{gustavoberned, aflorenzon\}@unipampa.edu.br
}

\section{Introdução}

A exploração do paralelismo em nível de threads (TLP - Thread Level Parallelism) tem sido amplamente utilizada para melhorar o desempenho de aplicações de diferentes domínios. Entretanto, muitas aplicações não escalam conforme o número de threads aumenta, ou seja, executar uma aplicação utilizando o máximo de threads não trará, necessariamente, o melhor resultado para tempo, energia ou o produto entre estas denominado EDP (Energy Delay Product), que visa medir a eficiência de consumo de energia de um circuito, devido a questões relacionadas à hardware e software [Raasch and Reinhardt 2003],[Lorenzon and Filho 2019].

Portanto, é preciso utilizar metodologias que consigam identificar um número ideal de threads para tais aplicações, sejam estas online (busca enquanto a aplicação é executada) ou offline (busca antes da execução da aplicação). Entretanto, metodologias online acabam adicionando uma sobrecarga na execução da aplicação, o que não acontece nas abordagens offline [Lorenzon et al. 2018]. Com base nisto, este trabalho apresenta uma metodologia genérica para reduzir significativamente o tempo de busca pelo número de threads ideal para aplicações paralelas que utilizam a metodologia offline, inferindo o ambiente de execução das aplicações paralelas utilizando apenas pequenos conjuntos de entrada de dados ${ }^{1}$.

\section{Reduzindo o Custo de Aprendizado de Estratégias Offline}

Este trabalho propõe um framework onde o usuário provê apenas uma aplicação paralela (e.g., binário) com um pequeno conjunto de entrada. O framework aplica um algoritmo de busca (fase de aprendizado) sobre esta pequena entrada para encontrar um número adequado de threads. Uma vez que este número é encontrado, a aplicação é executada (fase estável) com este número de threads sobre o conjunto de dados original.

$\mathrm{O}$ algoritmo utilizado durante a fase de aprendizado recebe como entrada um conjunto de núcleos $C$, um conjunto pequeno de entrada da aplicação $A$ e outros três parâmetros: $(i) \alpha$ número inicial de threads; $(i i) \beta$ taxa de aumento do número de threads; (iii) número máximo de núcleos $n$. O processo de aprendizado inicia aumentando exponencialmente o número de threads $\alpha$ a uma taxa $\beta$ até $n$, visando minimizar uma função de custo. Ao alcançar o máximo local, é realizado uma busca utilizando o método hill-climbing modificado, no intervalo onde o algoritmo encontrou o menor valor da função de custo. Para evitar mínimos locais e platôs durante este processo, o algoritmo

\footnotetext{
${ }^{1}$ Este estudo foi financiado pela FAPERGS - Códigos de Financiamento - 19/2551-0001224-1, 19/25510001689-1
} 
realiza movimentos laterais, onde, este ocorre antes de selecionar o número de threads ideal, testando as configurações vizinhas em outro ponto ainda não testado. Sendo assim, será considerado o melhor número de threads o conjunto que possua o menor valor na função de custo.

O framework proposto foi implementado na linguagem de programação Python3, sendo que, durante todo o processo aprendizado não há modificação e nem recompilação de código. Além do mais, o framework pode ser utilizado por qualquer uma das interfaces de programação paralela mais utilizadas, como OpenMP, MPI, Pthreads.

\section{Resultados Experimentais}

Dezoito aplicações paralelas já implementadas em $\mathrm{C}$ e $\mathrm{C}++$ foram executadas em dois ambientes multiprocessados: $(i)$ processador AMD 1700, contendo 8 núcleos e 16 threads com 16 GB de memória; (ii) processador Intel Core I9-7920X com 12 núcleos e 24 threads, dispondo de 64GB de memória. A metodologia proposta foi comparada com os seguintes cenários: (STD): execução da aplicação com o maior número de threads disponível no ambiente, sendo este o cenário padrão de execução, sem a intervenção do usuário; OMP_DYN: recurso presente no OPEN_MP que ajusta dinamicamente o número de threads para cada região paralela visando utilizar da melhor maneira possível os recursos presentes no sistema.

Os resultados mostram que, se avaliarmos o conjunto inteiro de benchmarks (média geométrica), a metodologia proposta obteve melhorias de $31 \%$ para EDP em relação a STD e de 35\% em relação ao OMP DYN. Além do mais, a metodologia proposta convergiu para o melhor número de threads para as aplicações ou muito próxima desta, quando comparada a busca sobre o pequeno conjunto de entrada ao maior, tendo um custo de aprendizado $88.3 \%$ e $82.6 \%$ menor nos processadores AMD e Intel respectivamente.

\section{Conclusão}

Apresentamos uma metodologia genérica que reduz significativamente o custo de busca pelo número ideal de threads em estratégias offline, inferindo o ambiente das aplicações utilizando pequenas entradas. Através de um grande conjunto de aplicações, mostramos que a metodologia consegue convergir para resultados ideais ou muito próximos. Como trabalhos futuros, a metodologia será aprimorada para identificar o número de threads para cada região paralela de uma aplicação.

\section{Referências}

Lorenzon, A. F. and Filho, A. C. S. B. (2019). Parallel Computing Hits the Power Wall - Principles, Challenges, and a Survey of Solutions. Springer Briefs in Computer Science. Springer.

Lorenzon, A. F., Oliveira, C. C. D., Souza, J. D., and Filho, A. C. S. B. (2018). Aurora: Seamless optimization of openmp applications. IEEE Transactions on Parallel and Distributed Systems, pages 1-15.

Raasch, S. E. and Reinhardt, S. K. (2003). The impact of resource partitioning on smt processors. In $P A C T$, pages 15-25. 